\title{
Psychostimulants, Brain Membrane Lipids and Dopamine Transmission
}

\section{Deborah J Luessen ${ }^{1}$ and Rong Chen ${ }^{1,2 *}$}

${ }^{1}$ Department of Physiology and Pharmacology, Wake Forest School of Medicine, Winston Salem, NC 27157, USA

${ }^{2}$ Center for the Neurobiology of Addiction Treatment, Winston Salem, NC 27157, USA

\begin{abstract}
Membrane lipids in the brain play important roles in regulation of the membrane compartmentalization, function and signaling of neurotransmitter transporters and receptors. This review summarizes findings on changes in the composition and metabolism of brain membrane lipids such as phospholipids, cholesterol and sphingolipids following chronic exposure to psychostimulants. We also discussed the mechanisms by which membrane lipids regulate the membrane compartmentalization and function of dopamine transports and receptors in animal brain tissues and cultured cell lines. This review indicates that chronic psychostimulant exposure causes the remodeling of brain membrane lipid, which may contribute to psychostimulant-induced functional alterations in dopamine transporters and receptors. Brain membrane lipids could be exploited as a new avenue for pharmacological interventions of abnormal brain dopamine transmission and dopamine-related addiction behavior.
\end{abstract}

Keywords: Brain membrane lipids; Lipid rafts; Psychostimulants; Dopamine transporters; Dopamine receptors

\section{Introduction}

Chronic abuse of psychostimulants remains a severe problem across the world, inflicting extensive physical, emotional and economic harm; however, there are still no effective treatments to date. Thus, there is an urgent need to better understand the brain mechanisms that underlie addiction so that new targets can be developed.

Chronic abuse of psychoactive drugs produces profound changes in neurotransmitter transporters and receptors, which have been associated with manifestations of many addiction phenotypes such as tolerance/sensitization, dependence and withdrawal (see reviews in [1]). Although the molecular mechanisms underlying psychostimulant-induced changes in transporters and receptors remain elusive, emerging evidence implicates brain lipids as potential mediators [2]. This review aims to provide a current understanding of the association between chronic psychostimulant exposure and brain membrane lipid profile and highlight the potential contribution of dysregulated brain membrane lipids to altered neurotransmission and associated addiction behavior. The impact of psychostimulant exposure on membrane phospholipid-derived intracellular lipid signaling was extensively reviewed elsewhere [3].

\section{Brain Membrane Lipids and Lipid Rafts}

The brain is the most lipid-rich organ in the human body [4]. The three major types of membrane lipids in the brain are phospholipids (e.g. glycerophospholipids), sphingolipids and cholesterol. Glycerophospholipids are glycerol-based phospholipids and a key structural component of the membrane. The brain contains many subtypes of glycerophospholipids including phosphatidylcholine, plasmalogens, phosphatidylserine, phosphatidylinositol, and phosphatidylethanolamine. Sphingolipids, another major lipid found in the plasma membrane, are synthesized from ceramide. The attachment of phosphocholine or saccharides to the hydroxyl group of ceramide leads to the production of sphingomyelin and glycosphingolipids. Gangliosides are glycosphingolipids with terminal sialic acids and are abundant in the brain. Cholesterol, a binding partner of sphingolipids, is a key sterol lipid and accounts for approximately $25 \%$ of all lipids in the brain [5]. The composition of these lipids is brain region- and cell type-dependent [6].
The cellular membrane is made of a lipid bilayer which is primarily composed of amphiphilic phospholipids. The lipid bilayer forms a physical barrier that separates the intracellular and extracellular environment. Additionally, sphingolipids and cholesterol form lipid rafts via hydrophobic interactions within the membrane (Figure 1). Lipid rafts are less fluid, when compared with the liquid crystalline phospholipid bilayer, and play a key role in organizing signaling molecules in the membrane to enhance or inhibit signaling events. Many G protein-coupled receptors (GPCRs) and associated signaling proteins are localized in cholesterol-enriched lipid raft microdomains [7]. Cholesterol within lipid rafts can interact with transmembrane proteins such as neurotransmitter receptors, transporters and ion channels. Thus, changes in the composition of the lipid rafts may therefore directly affect receptor compartmentalization, function, signaling and affinity for ligands.

The lipid composition of the membrane can be altered under various conditions such as stress, depression and anxiety via impaired lipid synthesis and metabolism [8]. Dysregulation of membrane lipid metabolism has been associated with a wide spectrum of neurological and psychiatric disorders including Alzheimer's disease, schizophrenia, Parkinson's disease and bipolar disorders [9-11]. This review focuses on abnormal membrane lipid profiles associated with chronic psychostimulant exposure, providing additional evidence to support the notion that brain lipids have an important function in neurotransmission.

\section{Psychostimulants and Brain Membrane Lipid Profile}

Psychostimulants, such as cocaine and methamphetamine, are highly addictive and widely abused drugs. Cocaine increases the

*Corresponding author: Rong Chen, Department of Physiology and Pharmacology, Wake Forest School of Medicine, Winston Salem, NC 27157, USA, Tel: 336-716-8605; Fax: 336-716-8501; E-mail: rchen@wakehealth.edu

Received May 09, 2016; Accepted May 28, 2016; Published June 06, 2016

Citation: Luessen DJ, Chen R (2016) Psychostimulants, Brain Membrane Lipids and Dopamine Transmission. J Biomol Res Ther 5: 143. doi:10.4172/2167 7956.1000143

Copyright: (c) 2016 Luessen DJ, et al. This is an open-access article distributed under the terms of the Creative Commons Attribution License, which permits unrestricted use, distribution, and reproduction in any medium, provided the original author and source are credited. 


\section{Extracellular Fluid}

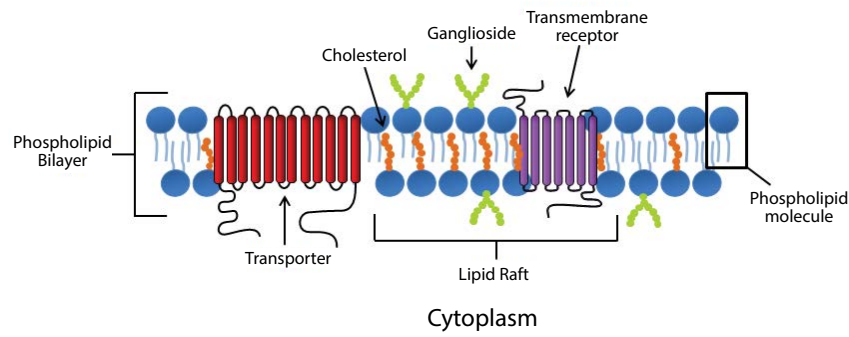

Figure 1 A schematic drawing of membrane lipid environment. Cellula membranes are organized into a phospholipid bilayer due to the unique chemical properties of the phospholipid molecule, including a hydrophilic head group and hydrophobic tail. The bilayer is interwoven with cholesterol molecules, which are particularly enriched in lipid raft microdomains, and serve many functions including regulating membrane fluidity and permeability and anchoring transmembrane proteins in the membrane. Lipid rafts are also enriched in other membrane lipids such as sphingolipids and play a multitude of roles, including regulation of signal transduction and protein trafficking. Membrane lipid composition is vital to proper structure and function of a diverse range of proteins; in particular, transmembrane proteins such as dopamine transporters and dopamine receptors.

extracellular levels of monoamines by blocking reuptake of dopamine, norepinephrine and serotonin through dopamine transporter (DAT), norepinephrine transporter (NET) and serotonin transporter (SERT), respectively. Treatment with methamphetamine, a substrate to DAT, NET and SERT, causes an accumulation of extracellular monoamines primarily via reverse transport of dopamine, norepinephrine and serotonin. The addictive property of psychostimulants is believed to be primarily associated with increased synaptic dopamine activity via acting on DAT [12]. Enhanced dopamine activity induced by cocaine or methamphetamine treatment results in altered membrane localization and function of DAT and dopamine receptors, which are associated with abnormal dopamine transmission and addiction behavior (see review in [13]). Although the mechanisms underlying psychostimulant-induced dysregulation of dopamine transporters and receptors remain elusive, scant evidence implicates the involvement of brain membrane lipids. Since brain membrane lipids play an integral role in membrane protein stability, signaling and function, alterations in membrane lipid composition likely induce functional changes to dopamine transporters/receptors and addiction behavior. Thus, it is important to understand the effects of psychostimulant exposure on membrane lipids in the brain.

\section{Phospholipids}

In the brain, phospholipids are the most abundant lipids [14]. Phospholipids comprise various molecular species with different fatty acid moieties in a brain region-dependent manner $[15,16]$. Chronic exposure to cocaine or methamphetamine has been shown to alter membrane phospholipid metabolism in a brain region-dependent manner. For example, repeated, intermittent injections of cocaine $(10-15 \mathrm{mg} / \mathrm{kg}$, i.p.) to male Sprague Dawley rats induce locomotor sensitization, which paralleled changes in brain phospholipids one week after the last cocaine injection [2]. Specifically, cocaine exposure increased phospholipid levels in the hippocampus and the ventral striatum. In contrast, cocaine reduced phospholipid levels in the cerebellum. Furthermore, cocaine-dependent poly drug abusers show reduced levels of phospholipids (e.g. phosphomonoester and phosphodiester) in the central white matter when compared to drug naïve controls [17]. Additionally, concurrent cocaine and heroin abusers show an increase in cerebral phosphomonoesters when compared to normal controls and no change in phosphodiesters [18]. The altered phospholipid metabolism likely resulted from the changes in the activity of brain phospholipid metabolic enzymes following psychostimulant exposure. Reduced activity of calciumstimulated phospholipase A2 (CPLA2) was reported in the striatum of human cocaine and methamphetamine users $[19,20]$. The reduced activity of cPLA2 may be associated with over-stimulation of striatal dopamine receptors because dopamine D2 receptors stimulate cPLA2 activity in CH101 cells [21]. Moreover, the activity of phosphocholine cytidylyltransferase, the rate-limiting enzyme of phosphatidylcholine synthesis, is also reduced in cocaine users [20]. Although the biological significance of each phospholipid species is largely unclear, it has been suggested that the cellular localization of phospholipid species is necessary for functional compartmentalization of the plasma membrane of neurons.

For example, reduced expression of 1-oleoyl-2-palmitoylphsphatidylcholine, a species of phospholipids and enriched at the protrusion tips of PC12 cells and presynaptic area of mouse neuronal synapses, abolishes the segregation of DAT and Gao proteins to the tip membrane [22]. Future studies should focus on understanding the significance of altered phospholipid species by psychostimulants in dopamine signaling. It is worth noting that phospholipids are abundant in the cortex and plays a critical role in cognition [14]. Thus, alterations in cortical membrane lipids may also be associated with cognition deficits in drug addicts.

\section{Cholesterol}

Brain cholesterol content can be altered by drugs of abuse. For example, chronic alcohol exposure increases membrane cholesterol content in whole guinea pig brain or rat cerebellar granule cultured cells $[23,24]$. However, the effect of psychostimulants on brain cholesterol content has yet to be directly measured. There is only one report on methylphenidate-mediated change in brain cholesterol level [25]. In this study, a single injection of methylphenidate to male Swiss mice reduced $[2-3 \mathrm{H}]$ acetate (a sterol precursor) incorporation into brain cholesterol during the 24 -hr period examined, suggesting that the pharmacological action of methylphenidate may involve lowering cholesterol content in the brain. Additionally, lower circulating cholesterol level was reported to be associated with cocaine relapse in a study of non-opiate and non-alcohol-dependent cocaine addicts, whose cholesterol levels had been determined once during their stay on an inpatient unit [26]. In this study, individuals with lower levels of circulating cholesterol had a significantly greater incidence of relapse 3 months, 6 months, and 1 year after discharge than those of patients who had higher levels of circulating cholesterol. Therefore, it is important to investigate the brain cholesterol content and metabolism following psychostimulant exposure, which may provide new avenues for pharmacological intervention of psychostimulant addiction.

\section{Sphingolipids and gangliosides}

Sphingolipids constitute a large class of lipids characterized by their eighteen carbon amino-alcohol backbones as sphingolipid bases. Modification in sphingolipid bases gives rise to diverse structure and function of sphingolipids. Although sphingolipids are not abundant compared to other membrane lipids in the brain, sphingolipids form lipid rafts with cholesterol within the membrane and play an important role in assembling GPCR signaling complex. The most abundant species of sphingolipids in mammalian cells is sphingomyelin [27]. Although there is no report of the effects of psychostimulants on sphingomyelin 
in vivo, a study using cultured Rat-1 fibroblasts indicates that cocaine treatment (250-500 $\mu \mathrm{M}, 2-3$ days) increases the content of sphingomyelin by abolishing sphingomyelinase activity [28], suggesting that cocaine modulates sphingomyelin content by regulating its metabolism. Additionally, limited data have shown alterations in brain gangliosides, a group of glycosphingolipids. For example, rat offspring exposed to cocaine in utero show elevated levels of total gangliosides and glycosphingolipids [29]. Moreover, chronic amphetamine treatment (IP, 20 days) modulates the ganglioside profile of rat frontal cortex, in which the level of quadrisialoganglioside is increased whereas GD1, GM2 and GM3 are decreased [30]. Importantly, altered membrane lipids are associated with cocaine and methamphetamine-induced behavior. For instance, repeated treatment with GM1 (30 mg/kg, IP for 7 days) attenuates amphetamine-induced locomotor sensitization in mice [31]. Moreover, pre-treatment with GM1 in rats enhances the rewarding property of cocaine as determined by conditioned place preference [32]. Although GM1 treatment does not alter the function and expression of DAT, it modifies the brain cocaine disposition by increasing brain cocaine levels. It has been shown that the levels of sphingolipids and gangliosides in the hippocampus and cortex are associated with depression and anxiety [8]. Cocaine addicts are often diagnosed with comorbid depression and/ or anxiety [33]. Thus, it is imperative to understand the consequence of altered sphingolipids and gangliosides on neurotransmission and associated behavioral manifestations.

\section{Psychostimulants, Lipid Rafts and Dopamine Transporters/Receptors}

Membranelipidraftsareenrichedin cholesteroland glycosphingolipids. The tight interaction between these lipids results in less fluid and highly ordered membrane structures that are resistant to detergent solubilization and are laterally wider than non-raft regions $[34,35]$. Membrane rafts not only control the localization of many GPCRs but also contain specific subsets of protein complexes that serve to compartmentalize cellular signaling processes. For example, rafts are associated with internalization and endocytotic cargo delivery [36], regulating the internalization of GPCRs and transporters. Emerging evidence indicates that psychostimulant exposure alters membrane compartmentalization (e.g. lipid rafts) of receptors in animal brains and cultured cells. For instance, repeated cocaine administration $(20 \mathrm{mg} / \mathrm{kg}$, i.p.) for seven days induces translocation of dopamine receptors in rat frontal cortex [37]. Moreover, dopamine D1 receptors (D1R) translocate from lipid raft to non-lipid raft and the cytoplasmic fractions of the frontal cortex following cocaine treatment whereas the localization of dopamine D5 receptors (D5R) and dopamine D2 receptors (D2R) were not significantly altered. Although there are no in vivo studies on the direct association between altered membrane lipid profile and receptor/transporter function, various studies using in vitro manipulations of membrane lipids especially cholesterol have provided compelling evidence of cholesterol-mediated localization, internalization and function of monoamine transporters and receptors. Lipid rafts are commonly disrupted in vitro with the treatment of methyl$\beta$-cyclodextrin $(\mathrm{M} \beta \mathrm{CD}, 2.5-10 \mathrm{mM}, 30 \mathrm{~min})$, which desorbs cholesterol from the membrane, or filipin $(0.5 \mathrm{mg} / \mathrm{ml}, 30 \mathrm{~min})$, which complexes with and functionally depletes cholesterol $[38,39]$. Literature suggests that the integrity of lipid rafts is critical for membrane compartmentalization and function of dopamine transporters and receptors.

\section{Cholesterol content and membrane compartmentalization of dopamine transporters and receptors}

DAT compartmentalization in membrane lipid raft and non-raft microdomains has been demonstrated in LLC-PK1 cells, neuroblastoma
N2A cells, and rat striatal tissues fractionated by sucrose density gradient centrifugation [40-42]. Both raft- and non-raft-associated DAT can undergo constitutive internalization in neuroblastoma SK$\mathrm{N}-\mathrm{MC}$ cells [43]. Disruption of lipid rafts with $\mathrm{M} \beta \mathrm{CD}$ or filipin in LLC$\mathrm{PK}_{1}$ cells does not alter the lateral movement of DAT between rafts and non-rafts [40]; however, treatment with M $\mathrm{CCD}(5 \mathrm{mM})$ or nystain $(25$ $\mu \mathrm{g} / \mathrm{ml}$, a cholesterol chelator in rafts) in HEK293 cells reduces DAT association with lipid raft microdomain [44]. There are conflicting data on the involvement of cholesterol in DAT internalization mediated by protein kinase $\mathrm{C}$ (PKC), a key mechanism of DAT internalization. Raft-associated DAT was shown to be resistant to internalization upon treatment with PKC, which is in contrast to non-raft-associated DAT in LLC-PK1 cells [40]. However, in SK-N-MC cells, PKC-stimulated DAT internalization primarily occurs in membrane raft microdomains [43]. The variations in cell lines and experimental techniques of these experiments may contribute to the discrepancy.

The regulation of DAT localization by membrane lipids is further supported by the formation of complexes between DAT and membrane raft-associated proteins. For example, flotillin-1 and DAT can be co-immunoprecipitated in HEK293 cells $[45,46]$. DAT and flotillin partition in similar detergent-resistant membrane fractions $[41,45]$ and depletion of flotillin shifts DAT localization to non-rafts [45]. Flotillin facilitates PKC-mediated DAT internalization in HEK293 cells [45]; however, it was also reported that flotillin knockdown via siRNA does not alter PKC-mediated DAT trafficking [46]. Additionally, DAT physically interacts with Rin, a Ras-like GTPase, and they form a complex in lipid raft microdomains of rat PC12 cells [47]. Disruption of Rin and DAT interaction abolishes PKC-regulated DAT internalization.Thus, DAT interactions with raft-associated proteins dictate the localization and/or internalization of DAT.

Membrane localization of dopamine receptors such as D1R and D2R is also mediated by membrane lipid composition (see review in [48]). D1Rs are primarily localized in lipid raft microdomains in HEK293 and COS-7 cells and can physically interact with caveolae, a raft-associated protein $[49,50]$. D1Rs and caveolae can form a functional complex in lipid rafts and regulate D1R-mediated G protein signaling. Similar to D1Rs, D2Rs are also primarily localized in lipid raft microdomains in HEK293 cells and mouse striatum tissues [51,52] although it is unknown whether D2Rs physically interact with certain raft-associated proteins. Moreover, dopamine-induced D2R internalization is largely restricted to D2Rs localized in lipid rafts [53]. Together, these data support the notion that the specialized compartmentalization of receptors/transporters in the lipid microdomains determines their internalization rate.

\section{Cholesterol content and the function of transporters/ receptors}

The localization of transporters/receptors in the lipid microdomains has functional significance. For instance, exogenously increasing membrane cholesterol level in striatal synaptosomes and neuronal-like MN9D cells increases the binding $B_{\text {max }}$ values for cocaine analogs but had no effect of DAT surface level [51]. Moreover, cholesterol depletion with $M B C D$ significantly reduced the ability of DAT to take up dopamine without altering DAT surface expression in LLC-PK1, HEK293 and N2A cells $[40,41,44]$. Furthermore, cholesterol depletion with $M \beta C D$ attenuates the ability of DAT to reverse transport dopamine in HEK293 and N2A cells $[41,44]$. Accordingly, knockdown of DAT-associated flottilin-1 proteins results in attenuation of amphetamine efflux in primary mesencephalic dopaminergic neurons, further demonstrating the importance of lipid raft localization in DAT function. 
Dopamine receptor function is also mediated by membrane lipid composition. For instance, D1R and D2R binding activity is primarily concentrated in the raft fractions of rat striatal membranes [52]. Additionally, the maximum number of D1R binding sites for $\mathrm{SCH} 23390$ in bovine caudate synaptosomes is insensitive to alterations in the ratio of cholesterol and phospholipid [54]. However, when the ratio is increased by $30 \%$, the binding affinity is reduced and the activity of dopamine-stimulated adenylate cyclase is markedly inhibited. In contrast, cholesterol depletion via $\mathrm{m} \beta \mathrm{CD}$ treatment increases D1Rmediated cAMP accumulation, which can be reversed upon cholesterol replenishment in COS-7 cells [50]. Moreover, co-expressing human D1R and lipid raft-associated protein caveolin-2 in HEK293 cells increases D1R-mediated adenylyl cyclase activity [49]. These findings suggest brain membrane lipids are key modulators of the membrane compartmentalization, trafficking and function of DAT and dopamine receptors. Dysregulation of brain membrane lipids would impact dopamine transporter/receptor-mediated dopamine transmission and associated addiction behavior.

\section{Therapeutic Potentials of Lipid-modifying Drugs in the Treatment of Psychostimulant Addiction}

Lipid-modifying drugs in recent years have been experimented for treatment of various neurological disorders including Alzheimer's disease; Parkinson's disease and stroke (see review in [55]). For example, there is an increasing interest in the therapeutic potential of reducing cholesterol levels in the treatment and prevention of Alzheimer's disease. A clinical trial on patients with mild to moderate Alzheimer's disease indicates that treatment with atorvastatin, a cholesterol lowering drug, produces positive outcomes on Alzheimer's progression after 6 and 12 month evaluation [56]. Moreover, a lower level of circulating cholesterol is associated with reduced production of amyloid beta observed in Alzheimer disease [57]. However, the beneficial effects of cholesterol lowering drugs have not been consistent [58]. Additionally, scant literature suggests that lipid-modifying drugs may be beneficial in the treatment of alcohol and nicotine abuse.

For example, intragastric administration of gemfibrozil $(50 \mathrm{mg} / \mathrm{kg})$, a cholesterol- and triglyceride-lowering drug, diminishes ethanol intake in male Sprague Dawley rats [59]. Furthermore, treatment with clofibrate (200-300 mg/kg, i.p.), another FDA approved cholesteroland triglyceride-reducing drug, significantly reduces nicotine-taking behavior and reinstatement in male Sprague Dawley rats with a prior history of nicotine exposure, which parallels clofibrate's ability to block nicotine-induced dopamine release in the nucleus accumbens and neuronal firing in the ventral tegmental area [60]. To date, there is no report on the therapeutics of lipid-modify drug in the treatment of psychostimulant addiction. These lipid-modifying drugs may be beneficial for drug addicts with abnormal lipid profiles.

\section{Conclusion}

Membrane lipids in the brain play an important role in regulation of the membrane compartmentalization, internalization and function of neurotransmitter transporters and receptors. This review highlights profound alterations in brain membrane lipids and paralleled changes in the function of dopamine transporters and receptors following chronic exposure to psychostimulants such as cocaine and methamphetamine. To date, it is unknown whether the remodeling of brain membrane lipids contributes to altered localization and function of dopamine transporters and receptors. Given the brain region dependent changes in membrane lipids, it is likely that these changes are associated with different aspects of addiction including enhanced motivation for drug- seeking and cognitive deficits. Thus, future studies designed to characterize the underlying mechanisms regulating the association between chronic psychostimulant abuse and membrane lipids, with respect to spatial and temporal aspects of dopaminergic neurotransmission, are essential. Brain membrane lipids could be exploited as a new avenue for pharmacological interventions of abnormal brain dopamine transmission and addiction behavior.

\section{Acknowledgment}

This work was supported by grants from National Institute of Drug Abuse and National Institute on Alcohol Abuse and Alcoholism (DA0006634 and T32 AA007565)

\section{References}

1. McCreary AC, Muller CP, Filip M (2015) Psychostimulants: basic and clinical pharmacology. Int Rev Neurobiol 120: 41-83.

2. Cummings BS, Pati S, Sahin S, Scholpa NE, Monian P, et al. (2015) Differential effects of cocaine exposure on the abundance of phospholipid species in rat brain and blood. Drug Alcohol Depend 152: 147-156

3. Orio L, Pavon FJ, Blanco E, Serrano A, Araos P, et al. (2013) Lipid transmitter signaling as a new target for treatment of cocaine addiction: new roles for acylethanolamides and lysophosphatidic acid. Curr Pharm Des 19: 7036-7049.

4. Dietschy JM, Turley SD (2004) Thematic review series: brain lipids. cholestero metabolism in the central nervous system during early development and in the mature animal. J Lipid Res 45: 1375-1397.

5. Yeagle PL (1985) Cholesterol and the cell membrane. Biochim Biophys Acta 822: $267-287$

6. van Meer G, Voelker DR, Feigenson GW (2008) Membrane lipids: where they are and how they behave. Nat Rev Mol Cell Biol 9: 112-124.

7. Allen JA, Halverson-Tamboli RA, Rasenick MM (2007) Lipid raft microdomains and neurotransmitter signalling. Nat Rev Neurosci 8: 128-140.

8. Muller CP, Reichel M, Muhle C, Rhein C, Gulbins E, et al. (2015) Brain membrane lipids in major depression and anxiety disorders. Biochim Biophys Acta 1851: 1052-1065.

9. Cheng D, Jenner AM, Shui G, Cheong WF, Mitchell TW, et al. (2011) Lipid pathway alterations in Parkinson's disease primary visual cortex. PLoS One 6: e17299

10. Chan RB, Oliveira TG, Cortes EP, Honig LS, Duff KE, et al. (2012) Comparative lipidomic analysis of mouse and human brain with Alzheimer disease. $\mathrm{J}$ Biol Chem 287: 2678-2688

11. Mazereeuw G, Herrmann N, Andreazza AC, Khan MM, Lanctot KL (2015) A meta- analysis of lipid peroxidation markers in major depression. Neuropsychiatr Dis Treat 11: 2479-2491.

12. Chen R, Tilley MR, Wei H, Zhou F, Zhou FM, et al. (2006) Abolished cocaine reward in mice with a cocaine-insensitive dopamine transporter. Proc Natl Acad Sci U S A 103: 9333-9338.

13. Howell LL, Kimmel HL (2008) Monoamine transporters and psychostimulant addiction. Biochem Pharmacol 75: 196-217.

14. Svennerholm L (1968) Distribution and fatty acid composition of phosphoglycerides in normal human brain. J Lipid Res 9: 570-579.

15. Zaima N, Hayasaka T, Goto-Inoue N, Setou M (2010) Matrix-assisted laser desorption/ionization imaging mass spectrometry. Int J Mol Sci 11: 5040-5055.

16. Chaurand P, Cornett DS, Angel PM, Caprioli RM (2011) From whole-body sections down to cellular level, multiscale imaging of phospholipids by MALDI mass spectrometry. Mol Cell Proteomics 10: 0110. 004259.

17. MacKay S, Meyerhoff DJ, Dillon WP, Weiner MW, Fein G (1993) Alteration of brain phospholipid metabolites in cocaine-dependent polysubstance abusers Biol Psychiatry 34: 261-264.

18. Christensen JD, Kaufman MJ, Levin JM, Mendelson JH, Holman BL, et al. (1996) Abnormal cerebral metabolism in polydrug abusers during early withdrawal: a 31P MR spectroscopy study. Magn Reson Med 35: 658-663.

19. Ross BM, Turenne SD (2002) Chronic cocaine administration reduces phospholipase $A(2)$ activity in rat brain striatum. Prostaglandins Leukot Essent Fatty Acids 66: 479-483. 
Citation: Luessen DJ, Chen R (2016) Psychostimulants, Brain Membrane Lipids and Dopamine Transmission. J Biomol Res Ther 5: 143. doi:10.4172/2167-7956.1000143

Page 5 of 5

20. Ross BM, Moszczynska A, Peretti FJ, Adams V, Schmunk GA, et al. (2002) Decreased activity of brain phospholipid metabolic enzymes in human users of cocaine and methamphetamine. Drug Alcohol Depend 67: 73-79.

21. Vial D, Piomelli D (1995) Dopamine D2 receptors potentiate arachidonate release via activation of cytosolic, arachidonate-specific phospholipase A2. J Neurochem 64: 2765-2772.

22. Kuge H, Akahori K, Yagyu K, Honke K (2014) Functional compartmentalization of the plasma membrane of neurons by a unique acyl chain composition of phospholipids. J Biol Chem 289: 26783-26793.

23. Bozhko G, Voloshin PV, Chursina VS (1988) Changes in cholesterol level and binding in guinea pig tissues during ethanol intoxication and cholesterolemia. Vopr Med Khim 34: 39-43.

24. Omodeo-Sale F, Pitto M, Masserini M, Palestini P (1995) Effects of chronic ethanol exposure on cultured cerebellar granule cells. Mol Chem Neuropathol 26: $159-169$

25. Charach G, Kaysar N, Grosskopf I, Rabinovich A, Weintraub M (2009) Methylphenidate has positive hypocholesterolemic and hypotriglyceridemic effects: new data. J Clin Pharmacol 49: 848-851.

26. Buydens-Branchey L, Branchey M (2003) Association between low plasma levels of cholesterol and relapse in cocaine addicts. Psychosom Med 65: 86-91.

27. Gault CR, Obeid LM, Hannun YA (2010) An overview of sphingolipid metabolism: from synthesis to breakdown. Advances in Experimental Medicine and Biology 688: 1-23.

28. Nassogne MC, Lizarraga C, N'Kuli F, Van Bambeke F, Van Binst R, et al (2004) Cocaine induces a mixed lysosomal lipidosis in cultured fibroblasts, by inactivation of acid sphingomyelinase and inhibition of phospholipase A1. Toxicol Appl Pharmacol 194: 101-110.

29. Leskawa KC, Jackson GH, Moody CA, Spear LP (1994) Cocaine exposure during pregnancy affects rat neonate and maternal brain glycosphingolipids. Brain Res Bull 33: 195-198.

30. Haselhorst U, Ghidoni R, Schenk H (1991) Changes of brain gangliosides in the frontal cortex of rats chronically treated with amphetamine, clozapine, haloperidol and ethanol. Biomed Biochim Acta 50: 931-935.

31. Bellot RG, Vital MA, Palermo-Neto J, Frussa-Filho R (1997) Repeated monosialoganglioside administration attenuates behavioral sensitization to amphetamine. Brain Res 747: 169-172.

32. Valdomero A, Hansen C, de Burgos NG, Cuadra GR, Orsingher OA (2010) GM1 ganglioside enhances the rewarding properties of cocaine in rats. Eur $J$ Pharmacol 630: 79-83.

33. Luthar SS, Glick M, Zigler E, Rounsaville BJ (1993) Social competence among cocaine abusers: moderating effects of comorbid diagnoses and gender. Am J Drug Alcohol Abuse 19: 283-298.

34. Brown DA, London E (2000) Structure and function of sphingolipid- and cholesterol- rich membrane rafts. J Biol Chem 275: 17221-17224.

35. Pike LJ (2003) Lipid rafts: bringing order to chaos. J Lipid Res 44: 655-667.

36. Parton RG, Richards AA (2003) Lipid rafts and caveolae as portals for endocytosis: new insights and common mechanisms. Traffic 4: 724-738.

37. Voulalas PJ, Schetz J, Undieh AS (2011) Differential subcellular distribution of rat brain dopamine receptors and subtype-specific redistribution induced by cocaine. Mol Cell Neurosci 46: 645-654.

38. Schnitzer JE, Oh P, Pinney E, Allard J (1994) Filipin-sensitive caveolaemediated transport in endothelium: reduced transcytosis, scavenge endocytosis, and capillary permeability of select macromolecules. J Cell Biol 127: $1217-1232$

39. Pike LJ (2006) Rafts defined: a report on the Keystone Symposium on Lipid Rafts and Cell Function. J Lipid Res 47: 1597-1598.

40. Foster JD, Adkins SD, Lever JR, Vaughan RA (2008) Phorbol ester induced trafficking-independent regulation and enhanced phosphorylation of the dopamine transporter associated with membrane rafts and cholesterol. J Neurochem 105: 1683-1699.

41. Adkins EM, Samuvel DJ, Fog JU, Eriksen J, Jayanthi LD, et al. (2007) Membrane mobility and microdomain association of the dopamine transporter studied with fluorescence correlation spectroscopy and fluorescence recovery after photobleaching. Biochemistry 46: 10484-10497.
42. Hong WC, Amara SG (2010) Membrane cholesterol modulates the outward facing conformation of the dopamine transporter and alters cocaine binding. $J$ Biol Chem 285: 32616-32626.

43. Gabriel LR, Wu S, Kearney P, Bellve KD, Standley C, et al. (2013) Dopamine transporter endocytic trafficking in striatal dopaminergic neurons: differential dependence on dynamin and the actin cytoskeleton. J Neurosci 33: 17836- 17846

44. Jones KT, Zhen J, Reith ME (2012) Importance of cholesterol in dopamine transporter function. J Neurochem 123: 700-715.

45. Cremona ML, Matthies HJ, Pau K, Bowton E, Speed N, et al. (2011) Flotillin-1 is essential for PKC-triggered endocytosis and membrane microdomain localization of DAT. Nat Neurosci 14: 469-477.

46. Sorkina T, Caltagarone J, Sorkin A (2013) Flotillins regulate membrane mobility of the dopamine transporter but are not required for its protein kinase C dependent endocytosis. Traffic 14: 709-724.

47. Navaroli DM, Stevens ZH, Uzelac Z, Gabriel L, King MJ, et al. (2011) The plasma membrane-associated GTPase Rin interacts with the dopamine transporter and is required for protein kinase C-regulated dopamine transporte trafficking. J Neurosci 31: 13758-13770.

48. Bjork K, Svenningsson P (2011) Modulation of monoamine receptors by adaptor proteins and lipid rafts: role in some effects of centrally acting drugs and therapeutic agents. Annu Rev Pharmacol Toxicol 51: 211-242.

49. Yu P, Yang Z, Jones JE, Wang Z, Owens SA, et al. (2004) D1 dopamine recepto signaling involves caveolin-2 in HEK-293 cells. Kidney Int 66: 2167-2180.

50. Kong MM, Hasbi A, Mattocks M, Fan T, O'Dowd BF, et al. (2007) Regulation of D1 dopamine receptor trafficking and signaling by caveolin-1. Mol Pharmacol 72: $1157-1170$.

51. Celver J, Sharma M, Kovoor A (2012) D(2)-Dopamine receptors target regulator of $\mathrm{G}$ protein signaling $9-2$ to detergent-resistant membrane fractions. J Neurochem 120: 56-69.

52. Vanderwerf SM, Buck DC, Wilmarth PA, Sears LM, David LL, et al. (2015) Role for Rab10 in Methamphetamine-Induced Behavior. PLoS One 10: e0136167.

53. Sharma M, Celver J, Octeau JC, Kovoor A (2013) Plasma membrane compartmentalization of D2 dopamine receptors. J Biol Chem 288: 1255412568.

54. Maguire PA, Druse MJ (1989) The influence of cholesterol on synaptic fluidity, dopamine D1 binding and dopamine-stimulated adenylate cyclase. Brain Res Bull 23: 69-74.

55. Bifulco M, Malfitano AM, Marasco G (2008) Potential therapeutic role of statins in neurological disorders. Expert Rev Neurother 8: 827-837.

56. Sparks DL, Sabbagh MN, Connor DJ, Lopez J, Launer LJ, et al. (2005) Atorvastatin for the treatment of mild to moderate Alzheimer disease: preliminary results. Arch Neurol 62: 753-757.

57. Puglielli L, Tanzi RE, Kovacs DM (2003) Alzheimer's disease: the cholesterol connection. Nat Neurosci 6: 345-351.

58. Eckert GP, Wood WG, Muller WE (2005) Statins: drugs for Alzheimer's disease? J Neural Transm (Vienna) 112: 1057-1071.

59. Barson JR, Karatayev O, Chang GQ, Johnson DF, Bocarsly ME, et al. (2009) Positive relationship between dietary fat, ethanol intake, triglycerides, and hypothalamic peptides: counteraction by lipid-lowering drugs. Alcohol 43: 433- 441

60. Panlilio LV, Justinova Z, Mascia P, Pistis M, Luchicchi A, et al. (2012) Novel use of a lipid-lowering fibrate medication to prevent nicotine reward and relapse: preclinical findings. Neuropsychopharmacology 37: 1838-1847. 